\title{
Observing movement
}

\section{Sophia Samuel}

\section{I feel the need to exercise to maintain my physical and mental wellbeing; it helps with my quality of life. I get exhausted pushing my body, but then I can sleep well at night.}

\section{- Tony Bienefelt}

This month's issue of Australian Journal of General Practice (AJGP) spotlights a common movement disorder in general practice: Parkinson's disease. Patients with Parkinson's disease, also a common neurodegenerative disorder, are managed by general practitioners (GPs) in all stages of illness. Importantly, Parkinson's disease is now recognised as a whole-body disorder, not a motor disorder with accompanying non-motor features. ${ }^{1,2}$

The motor signs of bradykinesia, resting tremor and rigidity are well known. But early motor symptoms, such as stiffness or weakness, may not even be reported, given the many input and output signals that govern movement. This highlights the challenge of early diagnosis and the endurance of formal gait assessments as a diagnostic tool.

The ability to walk independently requires complex neural and muscular control. Many GPs are skilled in the art of observation and incorporate informal assessments into their routines. GPs observe developmental milestones in the young, or early markers of disability in older adults. Clues may be visible when watching patients negotiate stairs, rise from chairs, walk to and from the consultation room, or turn and pass through doorways.

Unlike patients with more established Parkinson's disease, those presenting in the earlier course of their illness require careful focused examination for diagnosis.
Formal gait and postural assessments are complex because dysfunction is usually multifactorial. ${ }^{3,4}$ However, causes are mostly age specific, which assists in formulating age-appropriate differential diagnoses. After identifying an abnormal walking pattern in an older person, it is helpful to obtain collateral history from others in the household or from carers. In particular, the GP must consider whether the problems noted are temporary, contextual or truly persistent and progressive. Clearly this needs to be considered within the context of normal ageing. Similarly, there is a range of non-neurological causes to evaluate, including arthritis, medications, infections, and vascular and cardiorespiratory conditions, as all can result in movement difficulties.

Conceptualising Parkinson's disease as a whole-body condition influences the clinical process in two principal ways. First, the natural history of the disease is now understood to encompass more widespread consequences when compared with older concepts of diminished movement. Hence, as the average age of diagnosis is 60 years, when patients are employed or in active retirement, ${ }^{5}$ the psychosocial impact of the diagnosis may be more severe than in the past. Second, and as a consequence of the above, clinical care is far more focused on all aspects of the patient's wellbeing rather than prioritising only movement. Non-motor symptoms can be disabling and include gastrointestinal, mental health, sleep and autonomic nervous system dysfunction. As providers of whole-person healthcare, GPs are ideally situated to identify and manage these non-motor symptoms of Parkinson's disease as well as motor symptoms. Tools are available to help, such as the Non-Motor Symptom Questionnaire used to evaluate a patient across nine domains. ${ }^{6}$
Neurological disorders are often clinical diagnoses rather than imaging or laboratory-based diagnoses. As such, GPs are instrumental in diagnosis and ongoing clinical care. To support this important role, AJGP will be publishing a series of articles on neurology over the next few months. These articles will also focus on the clinical leadership of GPs within the multidisciplinary management team that may lead to significant improvements in quality of life.

In summary, our understanding of Parkinson's disease has changed from a motor disease to a whole-body disorder. GPs continue as always - in partnership with patients and their loved ones - to have crucial roles in early identification through to long-term clinical care.

\section{Author}

Sophia Samuel FRACGP, FARGP; Medical Editor, Australian Journal of General Practice; General Practitioner, Melbourne, Vic

\section{References}

1. Carroll V, Rossiter R, Blanchard D. Non-motor symptoms of Parkinson's disease. Aust J Gen Pract 2021;50(11):812-17.

2. Waller $S$, Williams $L$, Morales-Briceño $H$, Fung VSC. The initial diagnosis and management of Parkinson's disease. Aust J Gen Pract 2021;50(11):793-800.

3. Manickam A, Gardiner MD. Gait assessment in general practice. Aust J Gen Pract 2021;50(11):801-06.

4. Smythe A, Jivanjee M. The straight and narrow of posture: Current clinical concepts. Aust J Gen Pract 2021;50(11):807-10.

5. Tysnes OB, Storstein A. Epidemiology of Parkinson's disease. J Neural Transm (Vienna) 2017;124(8):901-05. doi: 10.1007/s00702-0171686-y.

6. International Parkinson and Movement Disorder Society. Non-Motor Symptom Questionnaire (NMSQ). Milwaukee, WI: International Parkinson and Movement Disorder Society, 2006. Available at www.movementdisorders.org/MDS/ MDS-Rating-Scales/Non-Motor-SymptomsQuestionnaire.htm [Accessed 12 October 2021].

correspondence ajgp@racgp.org.au 